\title{
ONO Tip Schiff Baz Komplekslerinin Sentezi, Karakterizasyonu, Katalitik Özellikleri ve Termal Çalışmaları
}

\author{
Hatice Gamze SOGUKOMEROGULLARI 1 (1), Mehmet SÖNMEZ ${ }^{* 2}$ (i) Gökhan CEYHAN ${ }^{3}$ (1) \\ ${ }^{1}$ Gaziantep Üniversitesi, Sağlı Hizmetleri MYO, Tıbbi Hizmetler ve Teknikleri Bölümü, 27310, Gaziantep, Türkiye \\ 2 Gaziantep Üniversitesi, Fen-Edebiyat Fakültesi, Kimya Bölümü, 27310, Gaziantep, Türkiye \\ ${ }^{3}$ Kahramanmaraș Sütçü İmam Üniversitesi, Kahramanmaraş MYO, Gıda Teknolojisi Bölümü, 46100, \\ Kahramanmaraş, Türkiye
}

(Alınış / Received: 25.01.2019, Kabul / Accepted: 12.09.2019, Online Yayınlanma / Published Online: 30.12.2019)

\section{Anahtar Kelimeler}

ONO tip Schiff baz kompleks, $\mathrm{N}$-aminopirimidin-2-on,

5-kloro-2-

hidroksiasetofenon,

Katalitik özellik
Özet: 5-kloro-2-hidroksiasetofenon ve 1-Amino-5-Benzoil-4-fenil-1H-pirimidin-2on'dan ( $N$-aminopirimidin-2-on) türeyen Schiff bazının bazı metal kompleksleri sentezlenmiştir. Bütün bileșikler elementel analiz, molar iletkenlik, manyetik moment ölçümü, IR, UV-Vis, kütle ve NMR (sadece Pd(II)) gibi spektral ve analitik tekniklerle karakterize edilmiştir. Pd(II) kompleksi kare düzlem geometriye sahipken, diğer kompleksler oktahedral yapıya sahiptir. Kompleksler iletkenlik özelliğine sahip değildir. Cu(II) ve Co(II) kompleksleri kısmi antiferromanyetik özellik gösterirken, Pd(II) kompleksi diyamanyetik özelliktedir. Schiff baz bileşiği ve yeni sentezlenen kompleksleri mikrodalga ışını altında siklohekzan oksidasyonu için incelenmiştir. Hem mikrodalga gücü hem de yeni katalizörlerin siklohekzanın $(\mathrm{CyH})$ siklohekzanon $(\mathrm{Cy}=\mathrm{O})$ ve siklohekzanole $(\mathrm{Cy}-\mathrm{OH})$ seçici yükseltgenmesini etkilediği görülmüștür. $\mathrm{Cu}(\mathrm{II})$ ve $\mathrm{Co}(\mathrm{II})$ komplekslerinin liganda ve diğer komplekslere oranla siklohekzanın oksidasyonu reaksiyonunda daha aktif oldukları bulunmuştur.

\section{Synthesis, Characterization, Catalytic Properties and Thermal Studies of ONO Type Schiff Base Complexes}

\section{Keywords}

ONO type Schiff base

complexes,

$\mathrm{N}$-aminopyrimidine-2-one,

5-chloro-2-

hydroxyacethophenone,

Catalytic properties

\begin{abstract}
Some metal complexes were synthesized from Schiff base derived from 5-chloro-2-hydroxyacethophenone and $\mathrm{N}$-aminopyrimidine-2-one. All compounds were characterized by elemental analyses, molar conductivity and magnetic measurements, IR, UV-Vis, Mass and NMR (for Pd (II)) spectral and analytic techniques. While $\mathrm{Pd}(\mathrm{II})$ complex displayed square planar geometry, the others exhibited octahedral geometry. The whole complexes showed non-electrolytic behavior. While $\mathrm{Cu}(\mathrm{II})$ and $\mathrm{Co}(\mathrm{II})$ complexes exhibited partial antiferromagnetic properties, Pd(II) complex showed diamagnetic property. Schiff base compound and new synthesized its some complexes were evaluated for cyclohexane oxidation under microwave irradiation. Both the microwave power and the novel catalysts were found to affect the selective oxidation of cyclohexane $(\mathrm{CyH})$ to cyclohexanone $(\mathrm{Cy}=\mathrm{O})$ and cyclohexanol $(\mathrm{Cy}-\mathrm{OH}) . \mathrm{Cu}(\mathrm{II})$ and $\mathrm{Co}(\mathrm{II})$ complexes were found to be more active in the reaction of the oxidation of cyclohexane than in the ligand and other complexes.
\end{abstract}

\section{Giriş}

Schiff bazları primer aminler ve karbonil bileşiklerinin kondenzasyonu yoluyla oluşan ve azometin bağı içeren bileşiklerdir. Schiff bazları ve komplekslerinin birçok farklı katalitik uygulamaları vardır. Bu katalitik uygulamalar arasında oksidasyon, olefinlerin aziridasyonu ve Kumada-Tamao-Corriu birleşme reaksiyonu gibi uygulamalar gösterilebilir
[1-3]. Literatür incelemesi yapıldığında genel olarak katalitik aktivite uygulamalarında metal komplekslerin ligandlara oranla çok daha aktif oldukları görülmektedir [4-7]. Bu da son zamanlarda metal komplekslerine olan ilgiyi artırmıștır. Araştırmalar Schiff baz komplekslerinin katalitik uygulamalarda seçici, etkili ve hatta enantiyoseçici özellikte olduklarını göstermektedir [8-10]. 
Pirimidinler DNA ve RNA yapısında bulunan, birçok önemli proseste rol alan ve farmakolojik öneme sahip olan yapılardır [11]. Pirimidinler ve türevi bileşiklerin birçok donör atomu bulunduğundan dolayı koordine olmaya eğilimli bileşiklerdir. Bu nedenle bu bileşikler metal komplekslerinin sentezinde kullanılmaktadır [12]. Bu çalıșmada 5kloro-2-hidroksiasetofenon ile $N$-aminopirimidin-2on bileşiğinin 1:1 kondenzasyonundan elde edilen Schiff baz ligandının Pd(II), Cu(II), Co(II) ve Ni(II) kompleksleri sentezlenmiștir. Sentezlenen bileșikler NMR, FT-IR, kütle, UV-Vis gibi çeşitli spektroskopik yöntemlerin yanı sıra elementel analiz, manyetik duyarlılık, elektrolitik iletkenlik ve TGA-DTA ile karakterize edilip, termal çalışmaları ve katalitik özelllikleri incelenmiştir. Sentezlenen yeni Schiff baz komplekslerinin katalitik çalışmaları mikrodalga ışınında siklohekzanın oksidasyonu üzerine yapilmiştır.

\section{Materyal ve Metot}

\subsection{Materyal}

Asetonitril, n-bütanol, dietileter, toluen, THF, EtOH, $\mathrm{MeOH}$, DMF, 5-kloro-2-hidroksiasetofenon, $\mathrm{Co}(\mathrm{AcO})_{2} \cdot 4 \mathrm{H}_{2} \mathrm{O}, \quad \mathrm{Cu}(\mathrm{AcO})_{2} \cdot \mathrm{H}_{2} \mathrm{O}, \quad \mathrm{Ni}(\mathrm{AcO})_{2} \cdot 4 \mathrm{H}_{2} \mathrm{O}$ ve $\mathrm{Pd}(\mathrm{AcO})_{2}$ gibi çalışmada kullanılan malzemeler Sigma-Aldrich ve Merck'in Türkiye'deki distribütör firmalarından sağlanmıştır. Tüm çözücüler kullanılmadan önce kurutulmuş ve saflaştırılmıştır. Başlangıç maddesi olan 1-Amino-5-benzoil-4-fenil1H-pirimidin-2-on ( $N$-aminopirimidin-2-on) literatüre uygun bir şekilde sentezlenmiștir $[13,14]$. Elementel analiz değerleri Thermo Scientific Flash 2000 marka ve modelli elemental analiz cihazıyla kaydedilmiştir. Schiff baz ligand ve komplekslerinin molar iletkenliği ise Thermo Scientific electron corporation model kondüktivimetre kullanılarak oda sıcaklığında ve DMF çözücüsünde belirlenmiştir. UVVis spektrumu PG Instruments T80+UV/Vis. Spektrometre ile ölçülmüştür. Örnekler DMF içinde çözülmüş ve spektrum 190-1100 nm arası taranmıștır. Komplekslerin manyetik moment değerleri Sherwood Scientific model cihazda ölçülmüştür. ${ }^{1} \mathrm{H}$ ve ${ }^{13} \mathrm{C}$ NMR spektrumları Bruker High Performance Digital FT-NMR (400 MHz) spektrometrede örnekler $\mathrm{d}_{6}$-DMSO'da çözülerek ve iç standart olarak TMS kullanılarak kaydedilmiştir. FTIR spektrumunda 4000-400 $\mathrm{cm}^{-1}$ aralığında Shimadzu FTIR (8000) model FT-IR spektrometresi kullanılmıştır. Termal davranışlar ise SetaramLabsys TGA/DTA model termal analiz cihazında $20-870{ }^{\circ} \mathrm{C}$ ve hava ortamında isıtılmıştır.

\subsection{Ligand sentezi (HL)}

1-[[1-(5-kloro-2-hidroksifenil)etiliden]amino]-4fenil-5-benzoil-pirimidin-2-on [HL] Schiff bazı literatüre göre sentezlenmiştir [11]. N aminopirimidin-2-on (0.291 g, $0.1 \mathrm{mmol}) 40 \mathrm{~mL} \mathrm{n}$ - bütanolde çözüldükten sonra üzerine 5-kloro-2hidroksiasetofenon ( $0.17 \mathrm{~g}, 0.1 \mathrm{mmol})$ ve katalizör olarak ise sodyum asetat eklendi. Reaksiyon karışımı 24 saat $100 \circ \mathrm{C}$ sıcaklıkta isıtıldı. Oda sicaklığına soğutulunca katı ürün süzüldü ve asetonitril-metanol (4:1) karışımında kristallendirildi [11].

\subsection{Komplekslerin genel sentezi}

$0.22 \mathrm{~g}$ (0.5 mmol) (HL) ligand $20 \mathrm{~mL}$ THF/MeOH (4:1) çözücü karışımında çözüldü. $0.25 \mathrm{mmol}$ $\mathrm{M}(\mathrm{AcO})_{2} \cdot \mathrm{nH}_{2} \mathrm{O}$ metal tuzu $10 \mathrm{~mL} \mathrm{MeOH'de} \mathrm{çözülerek}$ yavaşça ligandın üzerine eklendi. Karışım $65^{\circ} \mathrm{C}$ de 40 dakika boyunca karıștırıldı. Reaksiyon ürünü filtre edildi, soğuk metanolle yıkandı ve vakumlu desikatörde kurutuldu. [Cu(L)2], [Co(L)2], $\left[\mathrm{Ni}(\mathrm{L})_{2}\right] \cdot 5 \mathrm{H}_{2} \mathrm{O}$ ve $\left[\mathrm{Pd}(\mathrm{L})_{2}\right]$ kompleksleri sirasiyla $\mathrm{Cu}(\mathrm{AcO})_{2} \cdot \mathrm{H}_{2} \mathrm{O}(0.25 \mathrm{mmol}, 0.05 \mathrm{~g}), \mathrm{Co}(\mathrm{AcO})_{2} \cdot 4 \mathrm{H}_{2} \mathrm{O}$ $(0.25 \mathrm{mmol}, 0.063 \mathrm{~g}), \mathrm{Ni}(\mathrm{AcO})_{2} \cdot 4 \mathrm{H}_{2} \mathrm{O}(0.25 \mathrm{mmol}$, $0.062 \mathrm{~g})$ ve $\mathrm{Pd}(\mathrm{AcO})_{2}(0.25 \mathrm{mmol}, 0.056 \mathrm{~g})$ metal tuzları kullanılarak genel prosedürdeki gibi sentezlenmiştir. (Şekil 1 ve Şekil 2) Komplekslerin karakterizasyon dataları aşağıda verilmiştir.

[Cu(L)2]: [Verim: 0.094 g, 40\%] Kahverengi katı bileşik. $250^{\circ} \mathrm{C}$ üzeri dekompoze. Elementel Analiz: Hesaplanan: $\mathrm{C}_{50} \mathrm{H}_{34} \mathrm{Cl}_{2} \mathrm{CuN}_{6} \mathrm{O}_{6}$ (949.29): C, 63.26; $\mathrm{H}$, 3.61; N, 8.85. Bulunan: C, 62.80; H, 3.55; N, $8.93 \%$. IR / $\mathrm{cm}^{-1}:$ 3121m, 1715s, 1599s, 1362w, 534w, 434w, (s, kuvvetli; m, orta; w, zayıf). Meff: 1.61 BM. İletkenlik $2.2 \times 10^{-5} \mathrm{M}$ DMF $\left(\Lambda_{\mathrm{M}}\right): 0.59 \mu \mathrm{S} / \mathrm{cm}$. UV-Vis (DMF, nm, (Abs)): 400 (0.090), 330 (0.710), 298 (0.593), 294 (0.585). API-ES (MeOH): m/z 950.1[ $\left.\mathrm{Cu}+2(\mathrm{~L})+\mathrm{H}^{+}\right]$.

[Co(L)2]: [Verim: 0.22 g, 47\%] Açık kahverengi katı bileșik. $287^{\circ} \mathrm{C}$ üzeri dekompoze. Elementel Analiz: Hesaplanan: $\mathrm{C}_{50} \mathrm{H}_{34} \mathrm{Cl}_{2} \mathrm{CoN}_{6} \mathrm{O}_{6}$ (943.1): C, 63.57; $\mathrm{H}$, 3.63; N, 8.90. Bulunan: C, 63.95; H, 4.08; N, 9.19\%. IR / $\mathrm{cm}^{-1}:$ 3081m, 1688s, 1595s, 1355w; 536w; 443w, (s, kuvvetli; m, orta; w, zayıf). $\mu_{\text {eff: }} 1.87$ BM. İletkenlik $2.2 \times 10^{-5} \mathrm{M}$ DMF $\left(\Lambda_{\mathrm{M}}\right): 0.57 \mu \mathrm{S} / \mathrm{cm}$. UV-Vis (DMF, nm, (Abs)): 420 (0.080), 330 (0.667), 291(0.669). API-ES (MeOH): m/z 944.1 [Co + 2(L) + $\left.\mathrm{H}^{+}\right]$.

[Ni(L)2] $\mathbf{5} \mathbf{H}_{2}$ O: [Verim: $0.18 \mathrm{~g}, 34 \%$ ] Yeşil renkli katı bileșik. $267^{\circ} \mathrm{C}$ üzeri dekompoze. Elementel Analiz: Hesaplanan: $\mathrm{C}_{50} \mathrm{H}_{44} \mathrm{Cl}_{2} \mathrm{~N}_{6} \mathrm{NiO}_{11}(1032,2): \mathrm{C}, 58.05 ; \mathrm{H}$, 4.29; N, 8.12. Bulunan: C, 58.04; H, 4.16; N, $8.08 \%$. IR / cm-1: 3185m, 3060m, 1655s, 1612s, 1360w, 543w, 429w, (s, kuvvetli; m, orta; w, zayıf). $\mu_{\text {eff: }} 2.80$ BM. İletkenlik 2.2x10-5 M DMF $\left(\Lambda_{\mathrm{M}}\right): 0.41 \mu \mathrm{S} / \mathrm{cm}$. UV-Vis (DMF, nm, (Abs)): 430 (0.115), 330 (0.464), 285 (0.623). API-ES $(\mathrm{MeOH}): \mathrm{m} / \mathrm{z} 946.1\left[\mathrm{Ni}+2(\mathrm{~L})+2 \mathrm{H}^{+}\right]$.

[Pd(L)2]: [Verim: 0.25 g, 50\%] Kiremit kırmızısı renkli katı bileşik. $260^{\circ} \mathrm{C}$ üzeri dekompoze. Elementel Analiz: Hesaplanan: $\mathrm{C}_{50} \mathrm{H}_{34} \mathrm{Cl}_{2} \mathrm{~N}_{6} \mathrm{O}_{6} \mathrm{Pd}$ (990.17): C,60.53; H, 3.45; N, 8.47. Bulunan: C, 60.52; H, 3.51; N, $8.63 \%$. IR / $\mathrm{cm}^{-1}: 3100 \mathrm{~m}, 1689 \mathrm{~s}, 1603 \mathrm{~s}, 1349 \mathrm{w}$, 551w, 435w, (s, kuvvetli; m, orta; w, zayıf). $\mu_{\text {eff: }}$ diyamanyetik. İletkenlik $2.2 \times 10^{-5} \mathrm{M}$ DMF $\left(\Lambda_{\mathrm{M}}\right): 0.38$ 
$\mu \mathrm{S} / \mathrm{cm}$. UV-visible (DMF) [ $\left.\lambda_{\max } / \mathrm{nm},(\mathrm{Abs})\right]: 445$ (0.225), 310 (0.667), 266 (0.975). API-ES (MeOH): $\mathrm{m} / \mathrm{z} 992.1\left[\mathrm{Pd}+2(\mathrm{~L})+2 \mathrm{H}^{+}\right] .{ }^{1} \mathrm{H}$ NMR $\left(400 \mathrm{MHz}, \mathrm{d}_{6}-\right.$ DMSO) $\delta$ (ppm); s, singlet; d, dublet; m, multiplet: 9.97 (s, 2H, H-6), 7.61 (s, 4H, H-2', H-6'), 7.47 (d, 4H, $\left.J=7.28 \mathrm{~Hz}, \mathrm{H}-2^{\prime \prime}, \mathrm{H}-6^{\prime \prime}\right), 7.35\left(\mathrm{~d}_{\mathrm{d}}, 2 \mathrm{H}, \mathrm{H}-6^{\prime \prime \prime}\right) ; 7.21(\mathrm{~d}$, $\left.2 \mathrm{H}, J=7.28 \mathrm{~Hz}, \mathrm{H}-4^{\prime \prime \prime}\right), 7.07-7.17$ (m, 12H, Harm). 7.01 $\left(\mathrm{d}, 2 \mathrm{H}, \mathrm{J}=8.8 \mathrm{~Hz}, \mathrm{H}-3^{\prime \prime \prime}\right), 3.8\left(\mathrm{t}, 6 \mathrm{H}, \mathrm{CH}_{3}\right) .{ }^{13} \mathrm{C} \mathrm{NMR}\left(\mathrm{d}_{6}-\right.$ DMSO, ppm), $\delta 195.33 \quad(\mathrm{C}=0)_{\text {benzoil, }} 163.88$ $(\mathrm{C}=0)_{\text {pirimidin halka, }} 161.06(\mathrm{C}=\mathrm{N}), 146.27,139.76$, $134.64,131.41,130.38,130.01,129.11,128.27$, 127.75, 118.59, 116.68, 107.20 (aromatik karbonlar), $59.39\left(\mathrm{CH}_{3}\right)$.

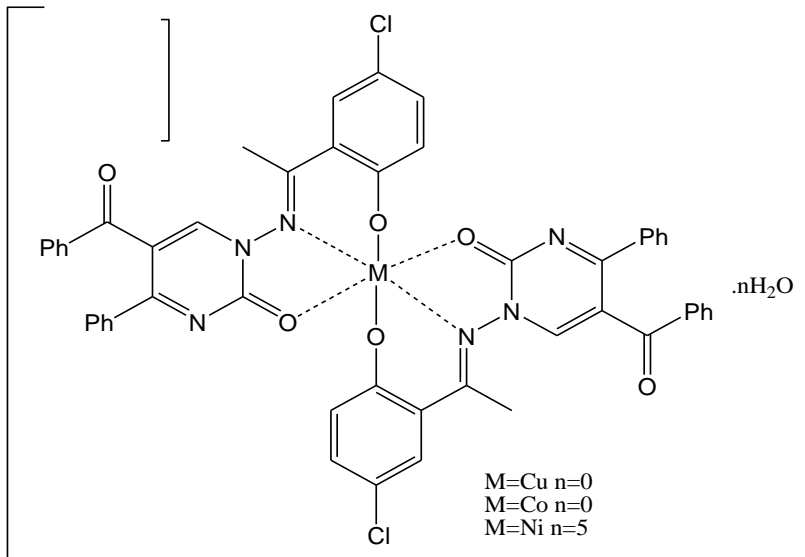

Şekil 1. HL ligandının $\mathrm{Cu}(\mathrm{II}), \mathrm{Co}(\mathrm{II})$ ve $\mathrm{Ni(II)}$ komplekslerinin önerilen yapısı

Mikrodalga ışın altında siklohekzanın katalitik oksidasyon çalışmalarında sentez tüpü içerisine katalizör (0.02 mmol), siklohekzan (2 mmol) ve $\mathrm{H}_{2} \mathrm{O}_{2}$ (4 mmol) koyulmuş ve 600 watt 'da 15 dakika boyunca mikrodalga ışınlarına maruz bırakılmıştır. Katalizör:substrat:oksidant oranı sırasıyla 1:100:200 şeklindedir [15]. Her oksidasyon denemesi için kompleksler $3 \mathrm{~mL}$ asetonitril içerisinde çözülmüş ve üzerine siklohekzan ve $\mathrm{H}_{2} \mathrm{O}_{2}$ eklenerek mikrodalga sentez tüpüne konmuştur. Sentez tüplerinin kapakları kapatıldıktan sonra, sentez tüpleri Berghof MWS3+ mikrodalga fırının içine yerleştirilmiş ve mikrodalga programı 15 dakika boyunca 600 watt gücünde uygulanmıştır. Sıcaklık $100^{\circ} \mathrm{C}$ 'ye ayarlanmış ve otomatik olarak kontrol edilmiștir. Çözücü ve substratın buharlaşması nedeniyle basınç 30 bar'a yükselmiştir. Daha sonra oksidasyon işlemini durdurmak için sentez tüplerine $\mathrm{H}_{2} \mathrm{O}(1.5 \mathrm{~mL})$ eklenmiştir. Organik asitler dışındaki yükseltgenme ürünleri olan organik bileşikler $5 \mathrm{~mL} \quad \mathrm{CH}_{2} \mathrm{Cl}_{2}$ ile ekstrakte edilerek ortamdan alınmıştır. $\mathrm{Bu}$ bileşikler GC ve GC-MS cihazıyla karakterize edilmiştir. Analizlerden önce hazırlanan harici kalibrasyon eğrilerinden $\mathrm{CyH}, \mathrm{Cy}-\mathrm{OH}$ ve $\mathrm{Cy}=\mathrm{O}$ miktarları hesaplanmıștır.

\section{Bulgular ve Tartışma}

Schiff baz kompleksleri ligand (HL) ve $\mathrm{Cu}(\mathrm{AcO})_{2} \cdot \mathrm{H}_{2} \mathrm{O}$, $\mathrm{Co}(\mathrm{AcO})_{2} \cdot 4 \mathrm{H}_{2} \mathrm{O}, \mathrm{Ni}(\mathrm{AcO})_{2} \cdot 4 \mathrm{H}_{2} \mathrm{O}$ ve $\mathrm{Pd}(\mathrm{AcO})_{2}$ metal tuzlarının 1:2 oranında gerçekleşen reaksiyonundan elde edilmiştir. Sentezlenen komplekslerin hepsi renkli, hava ortamında kararlı olan katı bileşiklerdir. Genel olarak THF, DMF, MeOH ve EtOH de çözünmektedir. Komplekslerin elementel analiz dataları incelendiğinde komplekslerin genel formülünün $\left[\mathrm{M}(\mathrm{L})_{2}\right] \cdot \mathrm{nH}_{2} \mathrm{O}$ olduğu görülmüştür. $\mathrm{Bu}$ formüldeki $\mathrm{L}$ HL ligandının anyonik formudur. Komplesklerin önerilen yapısı analitik datalarla oldukça uyum içerisindedir. Komplekslerin rengi, verimi, erime noktası, IR, UV-Vis ve manyetik moment sonuçları materyal metot kısmında ayrıntılarıyla belirtilmiştir. Komplekslerin DMF içerisinde $2 \times 10^{-5} \mathrm{M}$ konsantrasyondaki, molar iletkenlik değerleri ölçülmüştür. Bu değerler 0.59$1.87 \Omega^{-1} \quad \mathrm{~cm}^{2} \mathrm{~mol}^{-1}$ arasında kaydedilmiştir. $\mathrm{Bu}$ sonuçlara göre kompleksler elektrolitik iletkenliğe sahip değildir [16].

HL ligandının FT-IR spektrumu incelendiğinde 1609 $\mathrm{cm}^{-1}$ de karakteristik absorpsiyon bandı olan $v(\mathrm{C}=\mathrm{N})$ gerilmesi gözlenmektedir [17]. Komplekslerde bu karakteristik bandın yukarı $\left(9-18 \mathrm{~cm}^{-1}\right)$ ve aşağı (17 $\mathrm{cm}^{-1}$ ) kaydığı görülmektedir. $\mathrm{Bu}$ kaymalar kompleksleșmede metal iyonunun azometin azotunu kullandığını göstermektedir [18]. Ligandın FT-IR spektrumu incelendiğinde $3205 \mathrm{~cm}^{-1}$ de geniş bir $\mathrm{O}-\mathrm{H}$ gerilme bandı gözlenmektedir. $\mathrm{Bu}$ band komplekslerin tamamında kaybolmuştur. Tüm komplekslerin IR spektrumlarında $1342-1360 \mathrm{~cm}^{-1}$ fenolik bandın aşağı ve yukarı $\left(5-41 \mathrm{~cm}^{-1}\right)$ frekans kaydığı görülmüştür. $\mathrm{Bu}$ da fenolik $v(\mathrm{C}-\mathrm{O})$ grubun oksijen atomunun deprotone olup metal iyonuyla koordinasyona girdiğini akla getirmektedir [19]. HL ligandında $1649 \mathrm{~cm}^{-1}$ de görülen zayıf absorpsiyon bandı $v(\mathrm{C}=0)$ pirimidin gerilme titreșimine aittir $[11,20]$. Bu band $\mathrm{Cu}(\mathrm{II})$ ve $\mathrm{Co}(\mathrm{II})$ da görülmemekle beraber $\mathrm{Ni}(\mathrm{II})$ de $1657 \mathrm{~cm}^{-1} \mathrm{e}$ yüksek frekansa kaymıştır. $\mathrm{Bu}$ da pirimidin halkasına ait karbonil grubunun koordiasyona katıldığını göstermektedir [21]. Diğer taraftan Pd(II) kompleksinin IR spektrumu incelendiğinde pirimidin halkası üzerindeki karbonil grubuna ait $v(\mathrm{C}=0)$ titreşim bandının olduğu gibi durduğu gözlenmiştir. $\mathrm{Bu}$ da Pd(II) kompleksinin oluşumunda pirimidin üzerindeki karbonil grubunun koordinasyona katılmadığını göstermektedir [22]. Pd(II) kompleksi olușurken Schiff bazının fenolik oksijeni ve azometin grubunun azotu üzerinden bidentat olarak kompleksleşmeye girdiği düşünülmektedir (Şekil. 2). Komplekslerde 551-429 $\mathrm{cm}^{-1}$ aralıklarında yeni bandların oluştuğu görülmüştür. Yeni oluşan bantlar sırasıyla $v(\mathrm{M}-\mathrm{N})$ ve $v(\mathrm{M}-\mathrm{O})$ bantlarıdır $[17,18,23]$. $\mathrm{Ni}(\mathrm{II})$ kompleksindeki koordinasyon suyunun varlığ $3443 \mathrm{~cm}^{-1}$ deki geniş bir band, $750-800 \mathrm{~cm}^{-1}$ de düzlem içi sallanma ile $700-720 \mathrm{~cm}^{-1}$ de düzlem dışı sallanma titreşim bandları ile belirlenmiştir [23].

HL ligand ve Pd(II) kompleksinin ${ }^{1} \mathrm{H}-\mathrm{NMR}$ ve ${ }^{13} \mathrm{C}-\mathrm{NMR}$ spektrumları d6-DMSO çözücü kullanılarak alınmıştır. Diğer Ni(II), Co(II) ve $\mathrm{Cu}(\mathrm{II})$ kompleksleri paramanyetik özellikte olduklarından NMR spektrumları alınamamıştır. Ligandın ${ }^{1} \mathrm{H}-\mathrm{NMR}$ 
spektrumu incelendiğinde $\delta 10.68$ ppm'de singlet olarak fenolik proton [24], $\delta 9.97 \mathrm{ppm}$ de ise piridin halkasına ait proton singlet olarak görülmektedir. Liganda ait aromatik bölgedeki protonlar multiplet olarak 7.01-7.61 ppm arasında gözlemlenmiștir [11]. $\mathrm{Pd}(\mathrm{II})$ kompleksinin ${ }^{1} \mathrm{H}-\mathrm{NMR}$ ve ${ }^{13} \mathrm{C}$-NMR spektrumu incelendiğinde tek bir farkla ligandın sinyalleriyle yaklaşık olarak aynı yerlerde olduğu gözlemlenmiștir. Ligandda gözlenen fenolik proton Pd(II) kompleksinde kaybolmuştur (Şekil 3). Bu da fenolik protonun kompleksleşmede deprotone olarak koordinasyona katıldığının bir kanıtı olarak görülmektedir. Ligandın ${ }^{13} \mathrm{C}-\mathrm{NMR}$ spektrumu incelendiğinde 195 ppm, 163 ppm ve 161 ppm'de görülen piklerin sırasıyla $(C=0)$ grup $\left(\mathrm{C}_{7}\right),(\mathrm{C}=0)$ grup $\left(\mathrm{C}_{2}\right)$ ve $(\mathrm{C}=\mathrm{N})$ grubuna ait olduğu düşünülmektedir (Şekil 2) [25].

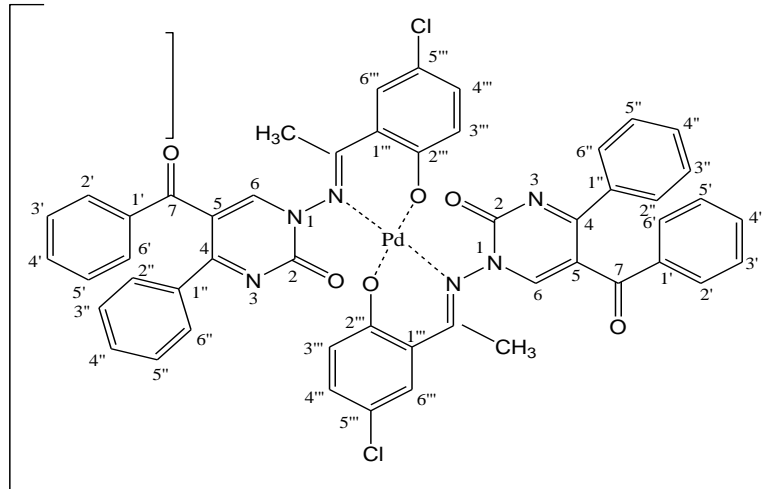

Şekil 2. Pd(II) kompleksinin önerilen yapısı

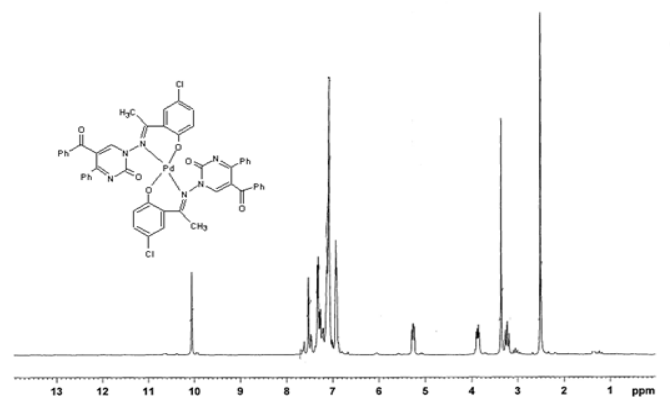

Şekil 3. Pd(II) kompleksinin ${ }^{1} \mathrm{H}-\mathrm{NMR}$ spektrumu

Schiff baz metal komplekslerinin kütle spektrumları incelendiğinde moleküler iyon piklerinin şu şekilde olduğu gözlenmiştir: m/z: $950.1[\mathrm{Cu}+2(\mathrm{~L})+1], \mathrm{m} / \mathrm{z}$ : $944.1[\mathrm{Co}+2(\mathrm{~L})+1], \mathrm{m} / \mathrm{z}: 946.1[\mathrm{Ni}+2(\mathrm{~L})+2], \mathrm{m} / \mathrm{z}$ : $992.1[\mathrm{Pd}+2(\mathrm{~L})] . \quad \mathrm{Pd}(\mathrm{II})$ ve Co(II) Schiff baz komplekslerinin kütle spektrumları Şekil 4 ve Șekil 5 olarak verilmiştir.

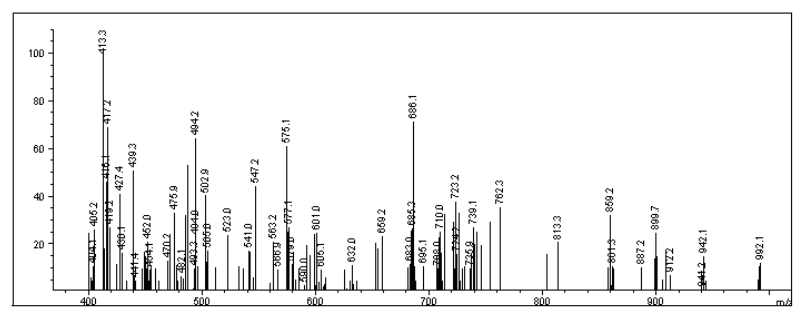

Şekil 4. Pd(II) kompleksinin API-ES kütle spektrumu

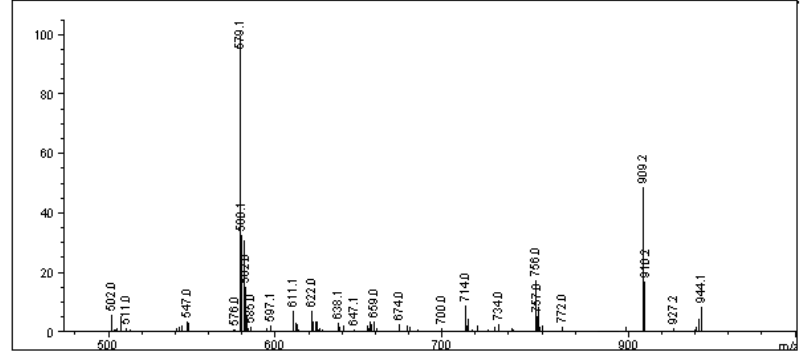

Şekil 5. Co(II) kompleksinin API-ES kütle spektrumu

Schiff baz ligand ve komplekslerinin elektronik absorpsiyon spektrumları DMF çözücüsü içerisinde alınmıştır. Materyal ve metot bölümünde ayrıntılarıyla datalar verilmiștir. 290-303 nm dalga boyları arasında aromatik halkalardaki $\pi \rightarrow \pi^{*}$ geçişlerine ait absorpsiyon pikleri görülmüştür [17, 23]. Ligandın UV-Vis spektrumunda $330 \mathrm{~nm}$ 'de $\mathrm{C}=\mathrm{N}$ imin grubuna ait $n \rightarrow \pi^{*}$ geçişlerinin olduğu gözlenmiştir. Kompleksleşme esnasında bu bandın așağı dalga boylarına kaydığı görülmüștür. $\mathrm{Bu}$ da komplekslerde metal iyonunun imin grubunun azotu üzerinden koordine olduğu düşüncesini güçlendirmektedir. Komplekslerin UV-Vis spektrumunda 400-445 nm arasında görülen absorbsiyon bandlarının (metalden liganda ya da liganddan metale) yük transfer bandları ve fenolat donör oksijeninin ortaklanmamış elektron çiftlerinden metal iyonlarına olan geçişler olduğu düșünülmektedir [18, 26]. Tüm komplekslerin elektronik spektrumlarında benzer geçişler gözlenmiştir. Dahası komplekslerde görünür bölgede liganddan metale ya da metalden liganda olan yük transfer geçişleri d-d geçişlerini maskelediği için bu geçişler belirlenememiştir.

Manyetik moment değerlerine bakıldığında, Co(II) komplesinin 1.87 BM manyetik moment değerine sahip olduğu, bu değerin oktahedral kompleksler için rapor edilen değerlere göre düşük olduğu görülmüștür [27, 28]. Bakır kompleksinin manyetik moment değeri 1.61 BM'dur. Bakır kompleksindeki ortaklaşmamış bir elektron nedeniyle bozuk oktahedral geometriye sahip olabileceği düşünülmektedir [29]. Ni(II) kompleksinin manyetik moment değeri 2.88 BM olarak ölçülmüştür ve kompleksin yapısının oktahedral olabileceği sonucuna varılmıştır [30]. Pd(II) kompleksinin elektronik spektrumunda ${ }^{1} \mathrm{~A}_{1 \mathrm{~g}} \rightarrow{ }^{1} \mathrm{~B}_{1 \mathrm{~g}}$ ve ${ }^{1} \mathrm{~A}_{1 \mathrm{~g}} \rightarrow{ }^{1} \mathrm{E}_{1 \mathrm{~g}}$ geçişlerinden dolayı 445 ve $310 \mathrm{~nm}$ 'de geçişler gözlemlenmiştir. $\mathrm{Bu}$ da yapının kare düzlem konfigürasyonda olduğunun bir işaretidir [31].

Termal analiz sonuçları analitik ve spektral datalarla oldukça uyum içerisindedir. Ni(II) kompleksinde 50 $80{ }^{\circ} \mathrm{C}$ 'de görülen kütle kaybı hidrasyon su moleküllerine aittir [32,33]. Metal komplekslerin termal kararlılıkları incelendiğinde sıralamanın şu şekilde olduğu görülür: $\mathrm{Ni}<\mathrm{Cu}<\mathrm{Pd}<\mathrm{Co}$ (Şekil 6, 7, 8, 9). Ni(II) kompleksinde termal bozunum dört basamakta gözlenmiştir. İlk basamakta 
dehidratasyon reaksiyonu, ikinci basamakta $\mathrm{HCl}$ çıkışı, üçüncü ve dördüncü basamakta ise organik grupların termal olarak bozunması ve en sonunda ilgili metal oksidine dönüşümü gözlenmiştir (Şekil 6). $\mathrm{Cu}(\mathrm{II})$ kompleksinin termal bozunma eğrisi incelendiğinde üç basamakta bozunmanın gerçekleştiği görülür. Birinci ve ikinci basamakta $\mathrm{HCl}$ çıkışları görülürken üçüncü basamakta organik gruplar dekompoze olarak ilgili metal oksidine dönüşmüştür (Şekil 7). Pd(II) kompleksinin de üç basamakta bozunduğu gözlenmiştir. Tıpkı $\mathrm{Cu}(\mathrm{II})$ kompleksinde olduğu gibi ilk iki basamakta $\mathrm{HCl}$ çıkışı gözlenirken üçüncü basamakta organik gruplar bozunarak dekompoze olmuş ve ilgili metal okside dönüşmüştür (Şekil 8). Co(II) kompleksinde termal bozunma iki basamakta gerçekleşmiştir. İki basamakta da organik grupların bozunarak ortamdan çıktığı ve ilgili metal oksidine dönüştüğü gözlenmiştir (Şekil 9).

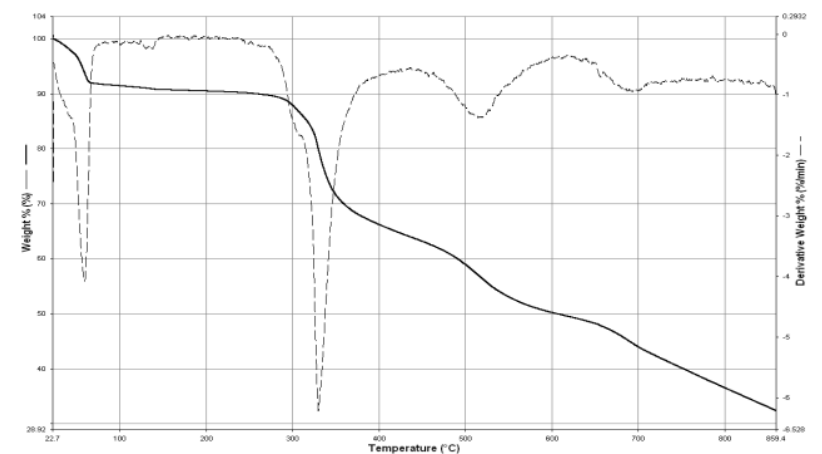

Şekil 6. Ni(II) kompleksinin TGA/DTA Eğrisi

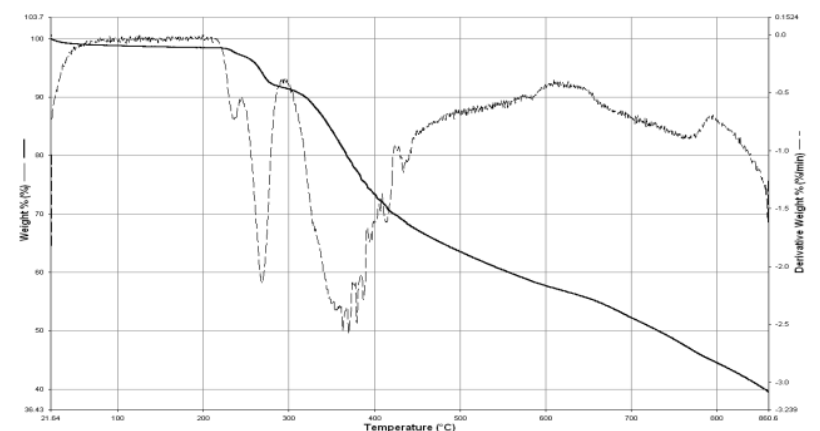

Şekil 7. Cu(II) kompleksinin TGA/DTA Eğrisi

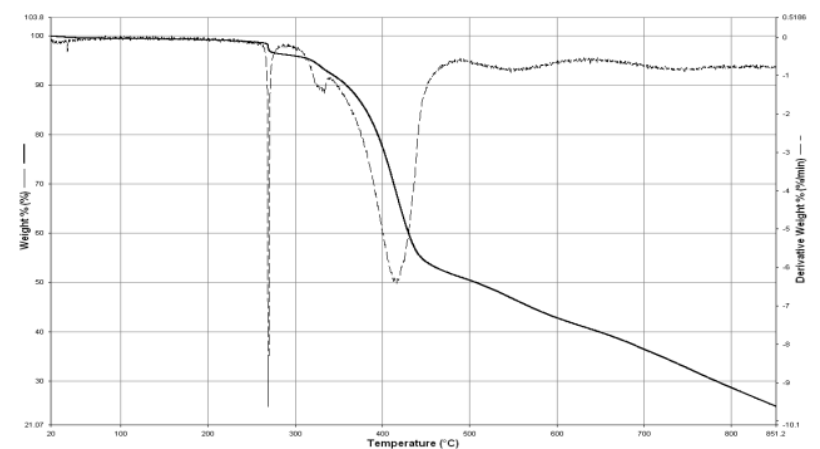

Şekil 8. Pd(II) kompleksinin TGA/DTA Eğrisi

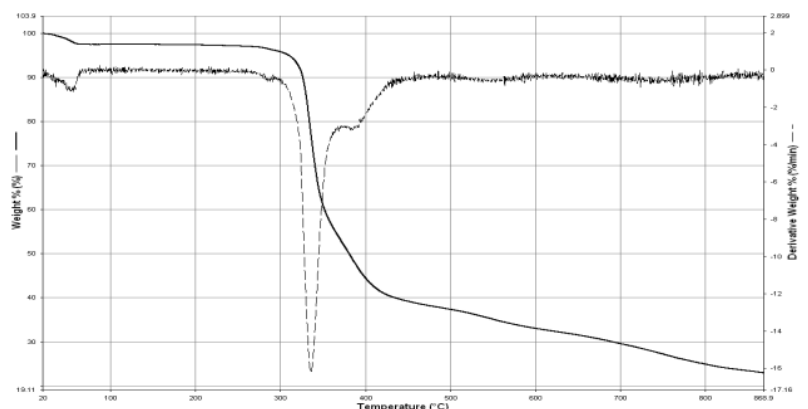

Şekil 9. Co(II) kompleksinin TGA/DTA Eğrisi

Bu kataliz reaksiyonunda ideal şartlar incelendiğinde katalizör: substrat: oksidant oranının 1:100:200 olduğu ve asetonitril çözücüsünde, $600 \mathrm{~W}$ mikrodalga gücünde ve 15 dakikada en etkili sonuca varıldığı görülmüștür. Sicaklık $100{ }^{\circ} \mathrm{C}$ 'de ve basınç 30 bar'da kontrollü olarak tutulmuştur. Katalizörsüz olarak da aynı şartlar altında çalışılmıştır [15]. Cu(II), Co(II) ve Ni(II) kompleksleri oktahedral yapıdayken, Pd(II) kompleksi kare düzlem geometriye sahiptir. Oksidasyon mekanizmasına bakıldığında ilk ve uzun olan basamak $\mathrm{CyH}$ 'nin $\mathrm{Cy}-\mathrm{OH}$ ve ondan $\mathrm{Cy}=\mathrm{O}$ ve diğer ileri oksidasyon ürünlerine yükseltgenmesi basamağıdır. Eğer ilk basamak kontrollü olarak yapılırsa, istenilen $\mathrm{Cy}-\mathrm{OH}$ ve $\mathrm{Cy}=\mathrm{O}$ ürünlerinin seçiciliklerindeki oran artırılabilir [34]. Bu reaksiyonda oluşabilecek ürünler Şekil 10'da gösterilmiştir. Bu çalışmada mikrodalga gücünün ve yeni katalizörlerin $\mathrm{CyH}^{\prime}$ nin $\mathrm{Cy}=\mathrm{O}$ ve $\mathrm{Cy}-\mathrm{OH}^{\prime}$ a seçici yükseltgenmesi reaksiyonunu etkileyebileceği gösterilmektedir. Mononükleer Cu(II) kompleksinin katalitik aktivitesinin $\mathrm{Ni}(\mathrm{II}), \mathrm{Pd}(\mathrm{II})$ ve $\mathrm{Co}(\mathrm{II})$ komplekslerine göre daha iyi olduğu gözlemlenmiștir. Literatüre bakıldığında Co(II) komplekslerinin katalitik aktivilerinin iyi olduğu görülmektedir. $\mathrm{Bu}$ çalışmada $\mathrm{Cu}(\mathrm{II})$ ve $\mathrm{Co}(\mathrm{II})$ komplekslerinin siklohekzan üzerinde yüksek katalitik aktiviteye sahip oldukları gözlenmiştir. Burada ligandın yapısının etkili olduğu düşülmektedir. Komplekslerde ligand yapısına ait olan gruplardaki $\pi-\pi^{*}$ elektron delokalizasyonunun siklohekzanı etkilediği düşünülmektedir. $\mathrm{Bu}$ durum oksidasyon reaksiyonunu etkiler. İstenilen $\mathrm{Cy}=\mathrm{O}$ ve $\mathrm{Cy}-\mathrm{OH}$ ürünlerinin eldesinde en iyi seçicilik $\mathrm{Cu}(\mathrm{II})$ kompleksine aittir. (Şekil 11). Mononükleer $\mathrm{Cu}(\mathrm{II})$ komplesinin katalitik aktivitesi daha önce rapor edilen komplekslerle kiyaslanabilir [35, 36]. Siklohekzanın yükseltgenme ürünlerine dönüşümünde $\quad \mathrm{Cu}(\mathrm{II})$-L'den $\quad \mathrm{Cu}(\mathrm{I})$-L'ye indirgenmektedir (L: Ligand). Cu(I)-L'ye indirgenme metal katyon etrafindaki ligand sayesinde kolaylaşmaktadır. $\mathrm{Bu}$ da yükseltgenme ürünleri $\mathrm{Cy}-$ $\mathrm{OH}$ ve $\mathrm{Cy}=0$ 'nun oluşumunda aktif bağların etkisini göstermektedir. Co(II) kompleksinin oktahedral geometriye sahip olması siklohekzan üzerindeki yüksek aktivitenin nedeni olabilir. Yükseltgenme ürünlerinin oluşumunda Co(II) komplekside yüksek aktiviteye sahiptir. 
Tablo 1. Tüm komplekslerin FTIR Verileri

\begin{tabular}{|c|c|c|c|c|c|c|c|c|}
\hline Bileşik & 0-H (s.b.) & $\begin{array}{l}\text { C-H } \\
\text { s.b. })\end{array} \quad$ (Ar & $\mathrm{C}=\mathbf{0}(\mathrm{s} . \mathrm{b})$ & C=N (s.b.) & C-O (s.b) & $\begin{array}{l}\text { C-Cl (Ar } \\
\text { s.b.) }\end{array}$ & M-N (s.b.) & M-O (s.b.) \\
\hline (HL) & 3205 & 3100 & 1686 & 1609 & 1342 & 1101 & - & - \\
\hline$\left[\mathrm{Cu}(\mathrm{L})_{2}\right]$ & - & 3121 & 1715 & 1599 & 1362 & 1098 & 534 & 434 \\
\hline [Co(L)2] & - & 3081 & 1688 & 1596 & 1355 & 1099 & 536 & 443 \\
\hline$\left[\mathrm{Ni}(\mathrm{L})_{2}\right]^{\cdot} \cdot 5 \mathrm{H}_{2} \mathrm{O}$ & 3185 & 3060 & 1655 & 1612 & 1318 & 1100 & 543 & 429 \\
\hline $\begin{array}{l}{\left[\mathbf{P d}(\mathbf{L})_{2}\right]} \\
\text { (s.b) gerilme ba } \\
\text { (Ar. s.b) Aroma }\end{array}$ & $\begin{array}{l}\text { - } \\
\text { ndı } \\
\text { ik gerilme b }\end{array}$ & 3100 & 1689 & 1603 & 1326 & 1095 & 551 & 435 \\
\hline
\end{tabular}

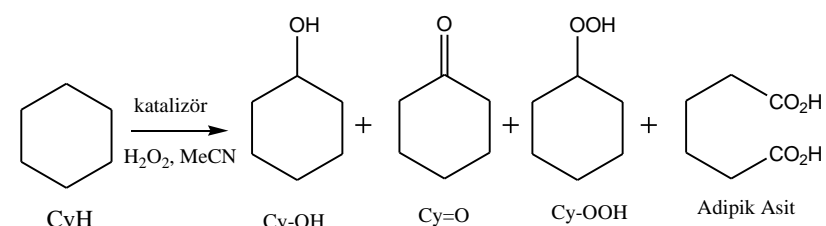

Sekil 10. Mikrodalga ıșın altında siklohekzanın katalitik oksidasyonu

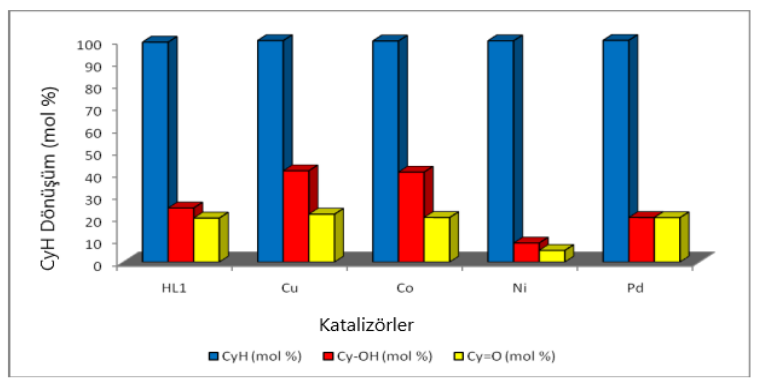

Şekil 11. Mikrodalga ışın altında siklohekzanın oksidasyonu üzerine komplekslerin etkileri. Her reaksiyonda $0.02 \mathrm{mmol}$ katalizör: $2 \mathrm{mmol}$ siklohekzan:4mmol hidrojen peroksit (1:100:200) ve $5 \mathrm{~mL}$ asetonitril kullanılmıștır. 600W güç 15 dakika boyunca uygulanmıştır. Reaksiyon sıcaklığı ve basıncı kapalı DAP60 kaplarında $100^{\circ} \mathrm{C}$ ve 30 bar'da tutulmuştur.

\section{Sonuç}

Bu çalışmada pirimidin halkası içeren dört yeni Schiff baz metal kompleksleri sentezlenmiş ve karakterize edilmiştir. Analitik datalar, UV-Vis ve FT-IR spektrumları manyetik moment ölçümleri $\mathrm{Cu}(\mathrm{II})$, Co(II) ve Ni(II) kompleksleri için uygun yapının oktahedral geometri olduğunu Pd(II) kompleksi için ise kare düzlem geometri olduğunu söylemektedir. Yeni sentezlenen Schiff baz komplekslerinin mikrodalga ışın altında siklohekzanın yükseltgenmesi üzerine katalitik çalışmaları yapılmıştır. Bu çalışmada hem mikrodalga gücün hem de yeni katalizörlerin CyH'nin $\mathrm{Cy}=\mathrm{O}$ ve $\mathrm{Cy}-\mathrm{OH}$ 'a seçici yükseltgenmesini etkilediği görülmüştür. Siklohekzanın yükseltgenmesi üzerine en yüksek katalitik aktiviteyi $\mathrm{Cu}(\mathrm{II})$ ve $\mathrm{Co}(\mathrm{II})$ kompleksleri göstermiştir.

\section{Teşekkür}

$\mathrm{Bu}$ araştırma Gaziantep Üniversitesi Bilimsel Araştırma Projeleri Birimi (FEF-10-09) tarafından desteklenmiştir.

\section{Kaynakça}

[1] Kalita, M., Gogoi, P., Barman, P., Sarma, B., Buragohain, A. K., Kalita, R. D. 2014. A new series of Ni (II), Cu (II), Co (II) and Pd (II) complexes with an ONS donor Schiff base: Synthesis, crystal structure, catalytic properties and bioactivities. Polyhedron, 74, 93-98.

[2] Khorshidifard, M., Rudbari, H. A., Askari, B., Sahihi, M., Farsani, M. R., Jalilian, F., Bruno, G. 2015. Cobalt (II), copper (II), zinc (II) and palladium (II) Schiff base complexes: Synthesis, characterization and catalytic performance in selective oxidation of sulfides using hydrogen peroxide under solvent-free conditions. Polyhedron, 95, 1-13.

[3] Bazarganipour, M., Salavati-Niasari, M. 2015. Grafting of copper(II) Schiff base complex on functionalized multi-wall carbon nanotubes: Synthesis, characterization and catalytic aziridination of olefins. Appl Catal A Gen, 502, 5764.

[4] Anitha, P., Manikandan, R., Vijayan, P., Prakash, G., Viswanathamurthi, P., Butcher, R.-J. 2015. Nickel(II) complexes containing ONS donor ligands: Synthesis, characterization, crystal structure and catalytic application towards C-C cross-coupling reactions. J Chem Sci, 127, 597608.

[5] Reddy, P. A. N., Datta, R., Chakravarty, A. R. 2000. Synthesis, X-ray structure and catalytic properties of a copper(II) Schiff base complex modeling the activity of the $\mathrm{Cu}_{\mathrm{B}}$ site of dopamine $\beta$-hydroxylase. Inorg Chem Commun, 3, 322-324.

[6] Dolaz, M., McKee, V., Uruş, S., Demir, N., Sabik, A. E., Gölcü, A., Tümer, M. 2010. Synthesis, structural characterization, catalytic, thermal and electrochemical investigations of bidentate Schiff base ligand and its metal complexes. Spectrochim Acta Part A, 76, 174-181.

[7] Tong, J., Li, Z., Xia, C. 2005. Highly efficient catalysts of chitosan-Schiff base Co(II) and Pd(II) complexes for aerobic oxidation of cyclohexane in the absence of reductants and solvents. J Mol Catal A Chem, 231, 197-203. 
[8] Fukuda, T., Katsuki, T. 1997. Highly enantioselective cyclopropanation of styrene derivatives using $\mathrm{Co}(\mathrm{III})$-salen complex as a catalyst. Tetrahedron, 53, 7201-7208.

[9] Masterson, D. S., Shirley, C., Glatzhofer, D. T. 2012. N-(4-[2.2] paracyclophanyl)-2'-

hydroxyacetophenone imine: An effective paracyclophane Schiff-base ligand for use in catalytic asymmetric cyclopropanation reactions. J Mol Catal A Chem, 361-362, 111-115.

[10] Genet, J. P., Juge, S., Achi, S., Mallart, S., Montes, J. R., Levif, G. 1988. Synthesis of $\alpha$-amino acids using transition metal catalysis - alkylation of schiff bases derived from $\alpha$-amimo acid esters (regio, stereo - selectivity). Tetrahedron, 44, 5263-5275.

[11] Soğukömeroğulları, H. G., Taşkın Tok, T., Yılmaz, F., Berber, İ., Sönmez, M. 2015. Synthesis, characterization, biological studies, and molecular modeling of mixed ligand bivalent metal complexes of Schiff bases based on $\mathrm{N}$ aminopyrimidine-2-one/2-thione. Turk J Chem, 39, 497-509.

[12] Sönmez, M., Sogukomerogullari, H. G, Öztemel, F., Berber, İ. 2014. Synthesis and biological evaluation of a novel ONS tridentate Schiff base bearing pyrimidine ring and some metal complexes. Med Chem Res, 23, 3451-3457.

[13] Akçamur, Y., Altural, B., Sarıpınar, E., Kollenz, G., Kappe, O., Peters, K., Peters, E., Schering, H. 1988. A convenient synthesis of functionalized 1H-pyrimidine-2-thiones. J Heterocyclic Chem, $25,1419-1422$.

[14] Akçamur, Y., Altural, B., Sarıpınar, E., Kollenz, G. 1989. A Simple Synthesis of Functionalized $1 \mathrm{H}-$ Pyrimidines.Reaction of Cyclic Oxalyl Compound. Monats für Chem, 120, 1015-1020.

[15] Ceyhan, G., Çelik, C., Uruş, S., Demirtaş, İ., Elmastaş, M., Tümer, M. 2011. Antioxidant, electrochemical, thermal, antimicrobial and alkane oxidation properties of tridentate Schiff base ligands and their metal complexes. Spectrochim Acta Part A, 81, 184-198.

[16] Emara, A. A. A., El-Sayed, B. A., Ahmed. E. A. E. 2008. Syntheses, spectroscopic characterization and thermal behavior on novelbinuclear transition metal complexes of hydrazones derived from4,6-diacetylresorcinol and oxalyldihydrazine. Spectrochim Acta Part A, 69, 757-769.

[17] Sönmez, M., Şekerci, M. 2007. The template synthesis, spectral characterization and thermal behavior of new binuclear Schiff base complexes derived from $\mathrm{N}$-aminopyrimidine with 2, 3butandion. J Serb Chem Soc, 72, 259-264.
[18] Belal, A. A. M., El-Deen, I. M., Farid, N. Y., Zakaria, R., Refat, M. S. 2015. Synthesis, spectroscopic, coordination and biological activities of some transition metal complexes containing ONO tridentate Schiff base ligand, Spectrochim Acta Part A, 149, 771-787.

[19] Sönmez, M., Berber, İ., Akbaș, E. 2006. Synthesis, antibacterial and antifungal activity of some new pyridazinone metal complexes. Eur J Med Chem, 41, 101-105.

[20] Önal, Z., Altural, B. 1999. Reactions of N-Aminopyrimidine Derivatives, with 1,3-Dicarbonyl Compounds. Turk J Chem, 23, 401-405.

[21] Abd-Elzaher, M. M. 2001. Spectroscopic characterization of some tetradentate Schiff bases and their complexes with nickel, copper and zinc. J Chin Chem Soc, 48, 153-158.

[22] Gülcan, M., Sönmez, M. Berber, İ. 2012. Synthesis, characterization, and antimicrobial activity of a new pyrimidine Schiff base and its $\mathrm{Cu}$ (II), Ni (II), Co (II), Pt (II), and Pd (II) complexes. Turk J Chem, 36, 189-200.

[23] Sönmez, M., Şekerci, M. 2003. Synthesis, Characterization and Thermal Investigation of Copper (II), Nickel (II), Cobalt (II) and Zinc (II) Complexes with 5-Benzoyl-1-(phenylmethylenamino)-4-phenyl1H-pyrimidine-2-thione. Synth React Inorg MetOrg Chem, 33, 1689-1700.

[24] Sönmez, M., Çelebi, M. Berber, İ. 2010. Synthesis, spectroscopic and biological studies on the new symmetric Schiff base derived from 2, 6diformyl-4-methylphenol with Naminopyrimidine. Eur J Med Chem, 45, 19351940.

[25] Tümer, M., Deligönül, N., Gölcü, A., Akgün, E., Dolaz, M., Demirelli, H., Dığrak, M. 2006. Mixedligand Copper (II) Complexes: Investigation of their Spectroscopic, Catalysis, Antimicrobial and Potentiometric Properties. Trans Met Chem, 31, 1-12.

[26] Ray, S., Konar, S., Jana, A., Das, K., Dhara, A., Chatterjee, S., Kar, S. K. 2014. Syntheses, crystal structure, spectroscopic and photoluminescence studies of mononuclear copper(II), manganese(II), cadmium(II), and a 1D polymeric $\mathrm{Cu}(\mathrm{II})$ complexes with a pyrimidine derived Schiff base ligand. J Mol Struct, 1058, 213-220.

[27] Gülcan, M., Sönmez, M. 2011. Synthesis and characterization of $\mathrm{Cu}$ (II), Ni (II), Co (II), Mn (II), and Cd (II) transition metal complexes of tridentate schiff base derived from o-vanillin and N-aminopyrimidine-2-thione. Phosphorus Sulfur and Silicon, 186, 1962-1971.

[28] Ekmekcioglu, P., Karabocek, N., Karabocek, S., Emirik, M. 2015. Synthesis, structural and 
biochemical activity studies of a new hexadentate Schiff base ligand and its $\mathrm{Cu}(\mathrm{II})$, Ni(II), and Co(II) complexes. J Mol Struct, 1099, 189-196.

[29] Modi, C. K. 2009. Synthesis, spectral investigation and thermal aspects of coordination polymeric chain assemblies of some transition metal ions with bis-pyrazolones. Spectrochim Acta Part A, 71, 1741-1748.

[30] Tyagi, P., Chandra, S., Saraswat, B. S. Sharma, D. 2015. Design, spectral characterization, DFT and biological studies of transition metal complexes of Schiff base derived from 2-aminobenzamide, pyrrole and furan aldehyde. Spectrochim. Acta Part A, 143, 1-11.

[31] Baran, T., Açlksöz, E., Mentes, A. 2015. Carboxymethyl chitosan Schiff base supported heterogeneous palladium (II) catalysts for Suzuki cross-coupling reaction. J Mol Catal A Chem, 407, 47-52.

[32] Tümer, M., Köksal, H., Serin, S. 1998. Synthesis, Characterization and Thermal Investigation of
Some Metal Complexes Derived from New Schiff Base Ligands. Synth React Inorg Met-Org Chem, 28, 1393-1404.

[33] Sönmez, M., Çelebi, M., Levent, A., Berber, İ., Şentürk, Z. 2010. A new pyrimidine-derived ligand, $\mathrm{N}$-pyrimidine oxalamic acid, and its $\mathrm{Cu}$ (II), Co (II), Mn (II), Ni (II), Zn (II), Cd (II), and Pd (II) complexes: synthesis, characterization, electrochemical properties, and biological activity. J Coord Chem, 63, 848-860.

[34] Urus, S., Dolaz, M., Tümer, M. 2010. Synthesis and Catalytic Activities of Silica-Supported Multifunctional Azo-Containing Schiff Base Complexes with $\mathrm{Cu}(\mathrm{II}), \mathrm{Co}(\mathrm{II}), \mathrm{Ni}(\mathrm{II})$ and $\mathrm{Mn}(\mathrm{II})$. J Inorg Organomet Polym, 20, 706-713.

[35] Retcher, B., Costa, J. S., Tang, J., Hage, R., Gamez, P., Reedijk, J. 2008. Unexpected high oxidation of cyclohexane by $\mathrm{Fe}$ salts and dihydrogen peroxide in acetonitrile. J Mol Catal A Chem, 286, 1-5.

[36] Luque, R., Badamali, S. K., Clark, J. H., Fleming, M., Macquarrie, D. J. 2008. Controlling selectivity in catalysis: Selective greener oxidation of cyclohexene under microwave conditions. Appl Catal A Gen, 341, 154-159. 